\title{
新型哌啶基肉桂醛缩氨基硫腿衍生物的设计、合成及抑菌活性研究
}

\author{
张学博马航宇孙腾达雷
杨新玲张晓鸣凌 云* \\ (中国农业大学理学院 应用化学系 北京 100193)
}

\begin{abstract}
摘要 为了发现高活性杀菌剂先导化合物, 利用骨架跃迁原理，以哌啶基苯甲醛缩氨基硫腿为先导化合物，设计并合 成了一系列未见文献报道的肉桂醮缩氨基硫腿类衍生物. 化合物结构经过 ${ }^{1} \mathrm{H} N M R 、{ }^{13} \mathrm{C} N M R$ 、IR、元素分析或 HRMS 确证. 离体抑菌活性测试结果表明, 一些化合物对多种病原真菌表现出优异的杀菌活性. 在 $50 \mu \mathrm{g} / \mathrm{mL}$ 浓度下, $N^{\prime}-[(1 E$, $2 E$ )-3-(4-氯苯基)烯丙基]哌啶-1-硫代酰胇(3a)和 $N^{\prime}-((1 E, 2 E)$-3-苯基烯丙基)哌啶-1-硫代酰肼(3p) 对苹果腐烂病菌、油菜 菌核病菌菌、瓜果腐霉病菌和水稻纹枯病菌的离体抑菌活性均在 $95 \%$ 以上. $\mathrm{EC}_{50}$ 测试结果表明, 化合物 $3 \mathbf{a}, 3 \mathbf{p}$ 对这 4 种病原真菌 $\mathrm{EC}_{50}$ 均在 $10 \mu \mathrm{g} / \mathrm{mL}$ 以内, 表现出广谱的杀菌活性. 初步构效关系分析表明, 将苯甲醛骨架替换为具有杀菌 活性的肉桂醛骨架结构, 有利于活性的提高.
\end{abstract}

关键词天然产物; 肉桂醛; 缩氨基硫艮; 合成; 杀菌活性

\section{Design, Synthesis and Fungicidal Activity of Novel Piperidine Containing Cinnamaldehyde Thiosemicarbazide Derivatives}

\author{
Zhang, Xuebo \\ Ma, Hangyu \\ Sun, Tengda \\ Lei, Peng \\ Yang, Xinling Zhang, Xiaoming Ling, Yun* \\ (Department of Applied Chemistry, College of Science, China Agricultural University, Beijing 100193)
}

\begin{abstract}
In order to find new lead compound with higher fungicidal activity, a series of novel cinnamaldehyde thiosemicarbazone derivatives were designed and synthesized according to scaffold hopping strategy, based on the lead of the thiosemicarbazide derivatives containing piperidine discovered in our preliminary studies. Their structures were confirmed by ${ }^{1} \mathrm{H}$ NMR, ${ }^{13} \mathrm{C}$ NMR, IR, elemental analysis or HRMS. The bioassay results indicated that some compounds exhibited excellent

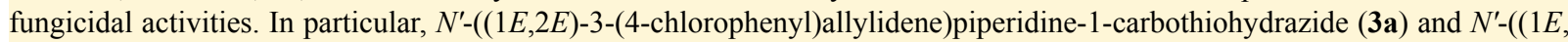
2E)-3-phenylallylidene)piperidine-1-carbothiohydrazide (3p) showed above $95 \%$ inhibitory rate against Cytospora $s p$, $S$. sclerotiorum, $P$. aphanidermatum and T. cucumeris at $50 \mu \mathrm{g} / \mathrm{mL}$ with $\mathrm{EC}_{50}$ values lower than $10 \mu \mathrm{g} / \mathrm{mL}$. The preliminary structure-activity relationship indicated that cinnamaldehyde contributes more to the bioactivity than benzaldehyde.

Keywords natural product; cinnamyl aldehyde; thiosemicarbazide; synthesis; fungicidal activity
\end{abstract}

农药的广泛使用, 极大地提高了粮食的产量与品 质. 但是，截止到目前为止，世界上仍然有约七分之一 的人口无法从充足的粮食中获得足够的能量和蛋白质. 同时，世界人口在不断地增加, 到 20 世纪中叶, 预计世 界人口将达到 90 亿. 因此, 新农药的研发对确保粮食安 全显得尤为重要 ${ }^{[1]}$. 天然产物在农药领域具有广泛的应 用 ${ }^{[2]}$, 嘧菌酯和吡唑醚菌酯是目前市场上销售额最大的 两个杀菌剂(图 1), 同属于丙烯酸酯类杀菌剂, 而该类杀
菌剂来源于天然产物, 有效成分主要从天然产物蘑菇中 分离得到 ${ }^{[3]}$. 在杀菌剂领域目前共有约 308 个商品化药 剂, 其中近一半直接来源于天然产物或天然产物衍生 物. 2014 年全球杀菌剂销售额约 185 亿美元，其中天然 产物或天然产物衍生物开发的杀菌剂占到整个销售额 的 $64 \%{ }^{[4]}$.

肉桂是一种天然的香料, 其主要成分是肉桂醛, 在 我国已经有 2000 多年的栽培历史. 肉桂醛广泛应用于

* Corresponding author. E-mail: 1yun@cau.edu.cn

Received March 17, 2019; revised April 18, 2019; published online May 21, 2019.

Project supported by the National Natural Science Foundation of China (No. 21672258).

国家自然科学基金(No. 21672258)资助项目. 
<smiles>CO/C=C(/C(=O)OC)c1ccccc1Oc1cc(Oc2ccccc2C#N)ncn1</smiles><smiles>COC(=O)N(OC)c1ccccc1COc1ccn(-c2ccc(Cl)cc2)n1</smiles>

图 1 部分天然源衍生物杀菌剂结构式

Figure 1 Structures of some fungicide come from natural products

香料、医药、化妆品、农药等领域, 具有抗癌、抗细菌、 抗病毒、酪氨酸酶抑制活性等多重生物活性 ${ }^{[5 \sim 10]}$. 尤其 在农药领域，肉桂醛具有杀菌 ${ }^{[11]}$ 、杀蚊幼虫 ${ }^{[12]}$ 等生物活 性. 目前市场上有多个含肉桂醛骨架结构的商品化药 剂, 例如烯肟菌酯(Enoxastrobin)、烯肟菌胺(Fenaminstrobin)、烯酰吗啉(Dimethomorph)和氟吗啉(Flumorph) 等(图 2).<smiles>CCOCCOCc1ccccc1C(=COC)C(=O)OC</smiles><smiles>CNC(=O)/C(=N/OC)c1ccccc1CO/N=C(C)/C=C/c1c(Cl)cccc1Cl</smiles><smiles>COc1ccc(/C(=C/C(=O)N2CCOCC2)c2ccc(Cl)cc2)cc1OC</smiles><smiles>COc1ccc(/C(=C/C(=O)N2CCOCC2)c2ccc(F)cc2)cc1OC</smiles>

图 2 部分含肉桂醛骨架结构的杀菌剂

Figure 2 Some fungicides containing cinnamaldehyde fragment

缩氨基硫脲类化合物广泛应用于医药、农药等领域, 具有抗癌、抗肿瘤、抗寄生虫、治疗阿尔茨海默氏病、 抑菌、抑制酪氨酸酶等多种生物活性 ${ }^{[13 ~ 16]}$. 本课题组对 苯甲醛缩氨基硫脲进行了多年研究 ${ }^{[17]}$, 并发现含哌啶 环的苯甲醛缩氨基硫嫝类化合物对苹果腐烂病菌和油 菜菌核病菌的抑菌效果明显 ${ }^{[18]}$. 为了开发出具有更好 杀菌活性的化合物, 我们以前期发现的哌啶基苯甲醛缩 氨基硫脲为先导，在保留哌啶基缩氨基硫脲结构片段基 础上, 采用骨架跃迁原理, 将苯甲醛替换为具有杀菌活 性的天然肉桂醛, 设计合成了一系列含哌啶基肉桂醛缩
氨基硫嫝类衍生物(图 3). 并测试其离体杀菌活性, 希望 发现更高杀菌活性的先导化合物.

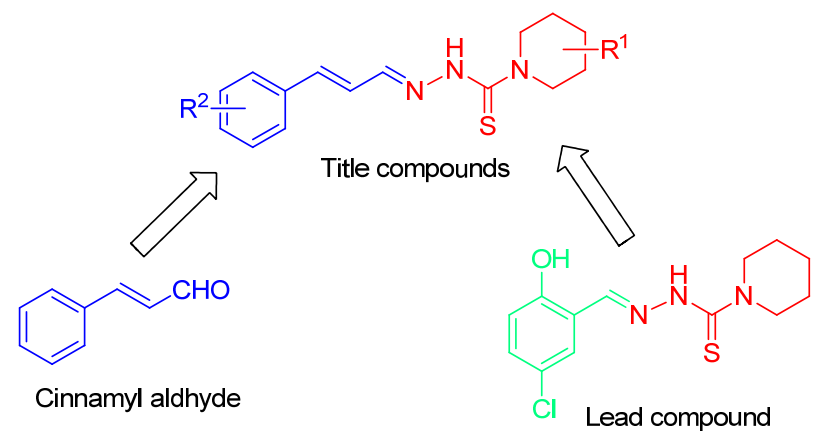

图 3 目标化合物的设计思想

Figure 3 Design strategy of the title compounds

\section{1 结果与讨论}

\section{1 目标化合物的合成}

中间体及目标化合物的合成路线如 Scheme 1 所示, 首先硫炭基二咪唑与取代哌啶发生亲核取代反应生成 中间体 $\mathbf{1}$, 再与水合肼反应得到关键中间体 2. 目标化合 物 3a 3p 通过中间体 2 与取代肉桂醛发生缩合反应得 到.

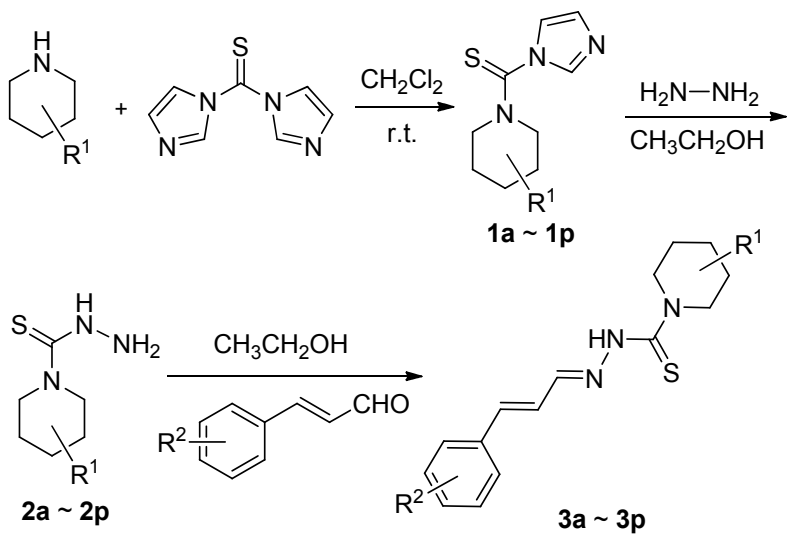

a: $\mathrm{R}^{1}=\mathrm{H}, \mathrm{R}^{2}=4-\mathrm{Cl} ; \mathbf{b}: \mathrm{R}^{1}=\mathrm{H}, \mathrm{R}^{2}=2-\mathrm{CH}_{3} ; \mathbf{c}: \mathrm{R}^{1}=\mathrm{H}, \mathrm{R}^{2}=2-\mathrm{OCH}_{3}$; d: $\mathrm{R}^{1}=\mathrm{H}, \mathrm{R}^{2}=4-\mathrm{OCH}_{3} ; \mathbf{e}: \mathrm{R}^{1}=\mathrm{H}, \mathrm{R}^{2}=4-\mathrm{NO}_{2} ; \mathbf{f}: \mathrm{R}^{1}=4-\mathrm{COOC}_{2} \mathrm{H}_{5}$, $\mathrm{R}^{2}=4-\mathrm{Cl} ; \mathbf{g}: \mathrm{R}^{1}=4-\mathrm{COOC}_{2} \mathrm{H}_{5}, \mathrm{R}^{2}=2-\mathrm{CH}_{3} ; \mathbf{h}: \mathrm{R}^{1}=4-\mathrm{COOC}_{2} \mathrm{H}_{5}$, $\mathrm{R}^{2}=2-\mathrm{OCH}_{3} ; \mathrm{i}: \mathrm{R}^{1}=4-\mathrm{COOC}_{2} \mathrm{H}_{5}, \mathrm{R}^{2}=4-\mathrm{OCH}_{3} ; \mathrm{j}: \mathrm{R}^{1}=4-\mathrm{COOC}_{2} \mathrm{H}_{5}$, $\mathrm{R}^{2}=4-\mathrm{NO}_{2} ; \mathbf{k}: \mathrm{R}^{1}=3-\mathrm{CH}_{3}, \mathrm{R}^{2}=4-\mathrm{Cl} ; \mathrm{I}: \mathrm{R}^{1}=3-\mathrm{CH}_{3}, \mathrm{R}^{2}=2-\mathrm{CH}_{3} ; \mathbf{m}:$ $\mathrm{R}^{1}=3-\mathrm{CH}_{3}, \mathrm{R}^{2}=2-\mathrm{OCH}_{3} ; \mathrm{n}: \mathrm{R}^{1}=3-\mathrm{CH}_{3}, \mathrm{R}^{2}=4-\mathrm{OCH}_{3} ; \mathbf{o}: \mathrm{R}^{1}=$ $3-\mathrm{CH}_{3}, \mathrm{R}^{2}=4-\mathrm{NO}_{2} ; \mathbf{p}: \mathrm{R}^{1}=\mathrm{H}, \mathrm{R}^{2}=\mathrm{H}$

图式 1 目标化合物的合成路线

Scheme 1 Synthetic route of target compounds

\section{2 谱图解析}

目标化合物结构均通过 ${ }^{1} \mathrm{H} N M R 、{ }^{13} \mathrm{C} N M R$ 、IR、 元素分析或 HR-ESI-MS 确证. 目标物的 IR 谱显示, $3200 \sim 3100 \mathrm{~cm}^{-1}$ 处为 $\mathrm{N}-\mathrm{H}$ 伸缩振动的吸收峰, 1700 $\mathrm{cm}^{-1}$ 附近为 $\mathrm{C}=\mathrm{S}$ 伸缩振动的吸收峰, $1650 \mathrm{~cm}^{-1}$ 附近为 
$\mathrm{C}=\mathrm{N}$ 伸缩振动吸收峰.

目标物的 ${ }^{1} \mathrm{H}$ NMR 谱显示, 化学位移在 $\delta 10.0 \sim$ 12.0 处的单峰为 $\mathrm{NH}$ 上的氢, 此处氢原子受到硫羰基和 $\mathrm{N}$ 原子的影响, 化学位移向低场移动; $\mathrm{N}=\mathrm{CH}$ 中的 $\mathrm{H}$ 受 $\mathrm{N}$ 原子吸电子作用的影响向低场移动, 化学位移在 $\delta$ $7.8 \sim 8.0$ 之间; 苯环上的氢位移在 $\delta 7.0 \sim 8.0$ 之间; $\mathrm{CH}=$ $\mathrm{CH}$ 化学位移受苯环上不同取代基的影响, 变化较大, 一般在 $\delta 6.0 \sim 8.0$ 之间; 哌啶环上与氮原子相连的 4 个 $\mathrm{H}$, 受 $\mathrm{N}$ 原子吸电子的影响向低场移动, 化学位移在 $\delta$ $3.0 \sim 5.0$ 之间.

\section{3 生物活性}

对 5 种植物病原真菌的初步离体抑菌活性试验, 结 果(表 1)表明, 在 $50 \mu \mathrm{g} / \mathrm{mL}$ 浓度下, 目标化合物对 5 种 供试菌株均表现出较明显的抑制效果. 其中化合物 3a、

3c、3f、3h、3k 和 $3 \mathrm{~m}$ 对水稻纹枯病菌的抑制活性表现 突出，抑菌率均为 $100 \%$; 化合物 $3 \mathbf{a} 、 3 \mathbf{d} 、 3 \mathbf{n}$ 和 $\mathbf{3 p}$ 对油 菜菌核病菌的抑制率在 $98 \%$ 以上; 化合物 $3 \mathbf{a} 、 3 \mathbf{3 b} 、 3 \mathbf{c} 、$

3d、3e、3h、3k、3m、3n、3o 和 3p 对苹果腐烂病菌抑 制率均在 $90 \%$ 以上. 以上化合物初步离体普篎抑菌活性 均优于先导化合物. 特别值得注意的是, 化合物 $\mathbf{3 a}$ 和 $3 \mathbf{p}$ 表现优异, 其普适性较广, 对苹果腐烂病菌、水稻纹 枯病菌、瓜果腐霉病菌和油菜菌核病菌 4 种真菌抑制率 均在 $95 \%$ 以上.

对普耖活性抑菌率在 $80 \%$ 以上的化合物进行离体 $\mathrm{EC}_{50}$ 活性测试, 结果(表 2 5)表明: 化合物 3a 对油菜菌
核病菌抑制活性 $\left(\mathrm{EC}_{50}=4.94 \mu \mathrm{g} / \mathrm{mL}\right)$ 优于商品化对照药 剂嘧菌酯 $\left(\mathrm{EC}_{50}=5.17 \mu \mathrm{g} / \mathrm{mL}\right)$ 及先导化合物 $\left(\mathrm{EC}_{50}=12.61\right.$ $\mu \mathrm{g} / \mathrm{mL})$. 化合物 3d 对瓜果腐霉病菌抑制活性 $\left(\mathrm{EC}_{50}=\right.$ $4.35 \mu \mathrm{g} / \mathrm{mL})$ 优于对照药剂嘧菌酯 $\left(\mathrm{EC}_{50}=16.88 \mu \mathrm{g} / \mathrm{mL}\right)$ 及先导化合物 $\left(\mathrm{EC}_{50}=5.37 \mu \mathrm{g} / \mathrm{mL}\right)$. 化合物 3a、3c、3f、 $3 \mathbf{h} 、 3 \mathbf{k} 、 3 \mathrm{~m}$ 和 $3 \mathbf{p}$ 对水稻纹枯病菌抑制活性 $\left(\mathrm{EC}_{50}\right.$ 均在 $5 \mu \mathrm{g} / \mathrm{mL}$ 以内)优于先导化合物 $\left(\mathrm{EC}_{50}=5.60 \mu \mathrm{g} / \mathrm{mL}\right)$, 但 不及对照药剂吡唑醚菌酯 $\left(\mathrm{EC}_{50}=0.03 \mu \mathrm{g} / \mathrm{mL}\right)$. 初步构 效关系分析表明: 哌啶环上电子效应对苹果腐烂病菌、 水稻纹枯病菌、油菜菌核病菌和瓜果腐霉病菌的抑菌活 性有重要影响, 呈现出 $\mathrm{R}^{1}$ 不含取代基或推电子基 $\left(\mathrm{R}^{1}=\right.$ $\left.\mathrm{H}, 3-\mathrm{CH}_{3}\right)>$ 吸电子基 $\left(\mathrm{R}^{1}=4-\mathrm{COOC}_{2} \mathrm{H}_{5}\right)$ 的规律; 苯环上 位置效应和电子效应对苹果腐烂病菌、水稻纹枯病菌和 油菜菌核病菌有重要影响, 呈现出苯环 2 位取代基 $\left(\mathrm{R}^{2}=2-\mathrm{OCH}_{3}\right)>4$ 位取代基 $\left(\mathrm{R}^{2}=4-\mathrm{OCH}_{3}\right)$, 苯环 $\mathrm{R}^{2}$ 无取 代基或吸电子取代基 $\left(\mathrm{R}^{2}=\mathrm{H}, 4-\mathrm{Cl}\right)>$ 给电子取代基 $\left(\mathrm{R}^{2}=4-\mathrm{OCH}_{3}\right)$ 的规律, 但随着苯环 $\mathrm{R}^{2}$ 取代基吸电子能 力增强, 抑菌活性降低 $\left(4-\mathrm{NO}_{2}<4-\mathrm{Cl}\right)$.

为讨论肉桂醛骨架代替苯甲醛骨架后化合物对杀 菌活性的影响, 本文选取活性最好的含肉桂醛骨架的化 合物 $3 a$ 和 $3 p$ 与对应的含苯甲醛骨架的化合物 $\mathbf{A}$ 和 $\mathbf{P}$ 进行杀菌活性对比(结构式见图 4). 生物活性测试结果 (表 2 5)表明: 化合物 3a 对瓜果腐霉病菌、油菜菌核病 菌、水稻纹枯病菌、苹果腐烂病菌的抑制活性 $\left(\mathrm{EC}_{50}\right.$ 分 别为 $9.04,4.94,2.35$ 和 $5.53 \mu \mathrm{g} / \mathrm{mL}$ ) 优于含氯取代苯甲醛

表 1 目标化合物 $3 \mathbf{a} \sim 3 \mathbf{3}$ 离体抑菌活性(抑制率/\% $)^{a}$

Table 1 Antifungal activity in vitro of target compounds $\mathbf{3 a} \sim \mathbf{3 i}$ (inhibitory rate/\%)

\begin{tabular}{|c|c|c|c|c|c|c|c|}
\hline Compd. & $\mathrm{R}^{1}$ & $\mathrm{R}^{2}$ & 瓜果腐霉病 & 水稻纹枯病 & 苹果腐烂病 & 油菜菌核病 & 水稻恶苗病 \\
\hline $3 a$ & $\mathrm{H}$ & $4-\mathrm{Cl}$ & 96 & 100 & 99 & 99 & 68 \\
\hline $3 \mathbf{b}$ & $\mathrm{H}$ & $2-\mathrm{CH}_{3}$ & 46 & 78 & 91 & 47 & 44 \\
\hline $3 \mathbf{c}$ & $\mathrm{H}$ & $2-\mathrm{OCH}_{3}$ & 93 & 100 & 100 & 70 & 63 \\
\hline 3d & $\mathrm{H}$ & $4-\mathrm{OCH}_{3}$ & 94 & 84 & 95 & 99 & 100 \\
\hline $3 e$ & $\mathrm{H}$ & $4-\mathrm{NO}_{2}$ & 80 & 85 & 95 & 66 & 53 \\
\hline $3 f$ & $4-\mathrm{COOC}_{2} \mathrm{H}_{5}$ & $4-\mathrm{Cl}$ & 92 & 100 & 78 & 59 & 49 \\
\hline $3 g$ & $4-\mathrm{COOC}_{2} \mathrm{H}_{5}$ & $2-\mathrm{CH}_{3}$ & 30 & 71 & 55 & 54 & 35 \\
\hline $3 h$ & $4-\mathrm{COOC}_{2} \mathrm{H}_{5}$ & $2-\mathrm{OCH}_{3}$ & 95 & 100 & 91 & 47 & 51 \\
\hline $3 \mathbf{i}$ & 4- $\mathrm{COOC}_{2} \mathrm{H}_{5}$ & $4-\mathrm{OCH}_{3}$ & 86 & 82 & 85 & 69 & 79 \\
\hline $3 \mathbf{j}$ & 4- $\mathrm{COOC}_{2} \mathrm{H}_{5}$ & $4-\mathrm{NO}_{2}$ & 82 & 88 & 80 & 51 & 73 \\
\hline $3 k$ & $3-\mathrm{CH}_{3}$ & $4-\mathrm{Cl}$ & 83 & 100 & 98 & 89 & 60 \\
\hline 31 & $3-\mathrm{CH}_{3}$ & $2-\mathrm{CH}_{3}$ & 31 & 76 & 80 & 42 & 45 \\
\hline $3 m$ & $3-\mathrm{CH}_{3}$ & $2-\mathrm{OCH}_{3}$ & 85 & 100 & 98 & 81 & 54 \\
\hline $3 n$ & $3-\mathrm{CH}_{3}$ & $4-\mathrm{OCH}_{3}$ & 84 & 81 & 95 & 98 & 94 \\
\hline 30 & $3-\mathrm{CH}_{3}$ & $4-\mathrm{NO}_{2}$ & 70 & 81 & 93 & 77 & 75 \\
\hline $3 p$ & $\mathrm{H}$ & $\mathrm{H}$ & 100 & 95 & 98 & 100 & 95 \\
\hline $\mathbf{A}$ & & & 44 & 100 & 91 & 62 & 49 \\
\hline $\mathbf{P}$ & & & 35 & 87 & 71 & 49 & 45 \\
\hline \multirow{2}{*}{\multicolumn{3}{|c|}{$\begin{array}{l}\text { Lead compound } \\
\text { 吡唑醚菌酯 }\end{array}$}} & 99 & 93 & 86 & 85 & 64 \\
\hline & & & 47 & 100 & 100 & 100 & 79 \\
\hline
\end{tabular}

${ }^{a}$ 浓度为 $50 \mu \mathrm{g} / \mathrm{mL}$. 
表 2 部分目标化合物对苹果腐烂病离体抑菌活性 $\mathrm{EC}_{50}$

Table $2 \mathrm{EC}_{50}$ of some target compounds against Valsa mali in vitro

\begin{tabular}{cccc}
\hline Compd. & $\mathrm{EC}_{50} /\left(\mu \mathrm{g} \cdot \mathrm{mL}^{-1}\right)$ & 线性方程 & $\mathrm{R}^{2}$ \\
\hline $\mathbf{3 a}$ & 5.53 & $y=2.2927 x+3.2971$ & 0.98 \\
$\mathbf{3 b}$ & 15.17 & $y=2.2541 x+2.3389$ & 0.97 \\
$\mathbf{3 c}$ & 8.56 & $y=1.7871 x+3.3347$ & 0.98 \\
$\mathbf{3 d}$ & 6.09 & $y=1.6526 x+3.7034$ & 0.95 \\
$\mathbf{3} \mathbf{3}$ & 17.60 & $y=0.9497 x+3.8172$ & 0.99 \\
$\mathbf{3 f}$ & 16.62 & $y=1.3833 x+3.3123$ & 0.97 \\
$\mathbf{3 h}$ & 14.23 & $y=1.3574 x+3.4357$ & 0.97 \\
$\mathbf{3 i}$ & 51.81 & $y=0.8067 x+3.6170$ & 0.91 \\
$\mathbf{3 j}$ & 24.72 & $y=1.2658 x+3.2367$ & 0.98 \\
$\mathbf{3 k}$ & 5.22 & $y=1.8442 x+3.6777$ & 0.99 \\
$\mathbf{3 1}$ & 8.64 & $y=1.8229 x+3.2947$ & 0.98 \\
$\mathbf{3 m}$ & 6.04 & $y=1.4877 x+3.8395$ & 0.98 \\
$\mathbf{3 n}$ & 33.53 & $y=0.9639 x+3.5296$ & 0.93 \\
$\mathbf{3 0}$ & 19.95 & $y=0.9814 x+3.7242$ & 0.96 \\
$\mathbf{3 p}$ & 5.20 & $y=2.0812 x+3.5097$ & 0.98 \\
Lead compound & 6.58 & $y=1.8537 x+3.4834$ & 0.99 \\
$\mathbf{A}$ & 5.70 & $y=1.3559 x+3.9746$ & 0.96 \\
$\mathbf{P}$ & 16.70 & $y=1.0628 x+3.7005$ & 0.94 \\
嘧菌酯 & 0.01 & $y=0.7677 x+6.4285$ & 0.96
\end{tabular}

表 3 部分目标化合物对水稻纹枯病离体抑菌活性 $\mathrm{EC}_{50}$

Table $3 \mathrm{EC}_{50}$ of some target compounds against Rhizoctonia solani in vitro

\begin{tabular}{cccc}
\hline Compd. & $\mathrm{EC}_{50} /\left(\mu \mathrm{g} \cdot \mathrm{mL}^{-1}\right)$ & 线性方程 & $\mathrm{R}^{2}$ \\
\hline $\mathbf{3 a}$ & 2.35 & $y=1.3663 x+4.4942$ & 0.96 \\
$\mathbf{3 c}$ & 2.20 & $y=0.9405 x+4.6784$ & 0.95 \\
$\mathbf{3 d}$ & 6.17 & $y=1.1077 x+4.1249$ & 0.97 \\
$\mathbf{3 e}$ & 6.80 & $y=1.1765 x+4.0206$ & 0.99 \\
$\mathbf{3 f}$ & 4.04 & $y=1.4348 x+4.1311$ & 0.97 \\
$\mathbf{3 h}$ & 3.66 & $y=1.2256 x+4.3109$ & 0.98 \\
$\mathbf{3 i}$ & 7.77 & $y=1.2411 x+3.8950$ & 0.95 \\
$\mathbf{3 j}$ & 8.60 & $y=1.5194 x+3.5798$ & 0.99 \\
$\mathbf{3 k}$ & 1.22 & $y=0.9178 x+4.9219$ & 0.95 \\
$\mathbf{3} \mathbf{3}$ & 1.46 & $y=1.1335 x+4.8152$ & 0.99 \\
$\mathbf{3 n}$ & 10.49 & $y=1.1615 x+3.8146$ & 0.94 \\
$\mathbf{3 o}$ & 10.22 & $y=1.2466 x+3.7418$ & 0.95 \\
$\mathbf{3 p}$ & 1.02 & $y=0.9761 x+4.9881$ & 0.98 \\
Lead compound & 5.60 & $y=0.6868 x+4.4864$ & 0.91 \\
$\mathbf{A}$ & 3.12 & $y=1.3133 x+4.3346$ & 0.92 \\
$\mathbf{P}$ & 5.60 & $y=0.9895 x+4.2629$ & 0.91 \\
\hline
\end{tabular}

结构的化合物 $\mathbf{A}\left(\mathrm{EC}_{50}\right.$ 分别为 $50.00,24.21,3.12,5.70$ $\mu \mathrm{g} / \mathrm{mL})$; 同样化合物 3p 对 4 种测试病原菌的抑制活性 优于含苯甲醛结构的化合物 $\mathbf{P}$. 初步表明肉桂醛骨架结 构的引入有利于提高化合物杀菌活性及杀菌谱. 同时我 们将化合物 3a (含 4-氯肉桂醛骨架)对水稻纹枯病菌、苹 果腐烂病菌的抑制活性与前期工作中 ${ }^{[18]}$ 含卤素苯甲醛 结构的化合物 B、C 和 D 进行对比(图 5), 发现化合物 3a 同样具有更高的活性, 进一步说明肉桂醛骨架结构 的引入有利于提高化合物杀菌活性.

\section{2 结论}

利用骨架跃迁原理，设计并合成了 15 个未见文献 报道的哌啶基肉桂醛缩氨基硫脲类衍生物. 目标化合物 均通过 ${ }^{1} \mathrm{H} N M R 、{ }^{13} \mathrm{C} N M R$ 、IR、元素分析或高分辨质 谱的确证. 生物活性测试结果显示, 所有目标化合物均 对 5 种病原真菌表现出明显的抑制活性. 其中化合物 3a、3p 对苹果腐烂病菌, 水稻纹枯病菌, 瓜果腐霉病菌 和油菜菌核病菌 4 种病原真菌 $\mathrm{EC}_{50}$ 均在 $10 \mu \mathrm{g} / \mathrm{mL}$ 以内, 
表 4 部分目标化合物对油菜菌核病离体抑菌活性 $\mathrm{EC}_{50}$

Table $4 \mathrm{EC}_{50}$ of some target compounds against Sclerotinia sclerotiorum in vitro

\begin{tabular}{|c|c|c|c|}
\hline Compd. & $\mathrm{EC}_{50} /\left(\mu \mathrm{g} \cdot \mathrm{mL}^{-1}\right)$ & 线性方程 & $\mathrm{R}^{2}$ \\
\hline $3 \mathbf{a}$ & 4.94 & $y=0.8204 x+4.4318$ & 0.91 \\
\hline 3d & 7.41 & $y=2.1292 x+3.1484$ & 0.91 \\
\hline $3 \mathbf{k}$ & 7.55 & $y=1.0553 x+4.0749$ & 0.99 \\
\hline $3 \mathrm{~m}$ & 9.57 & $y=1.3686 x+3.6580$ & 0.94 \\
\hline $3 n$ & 47.43 & $y=1.1461 x+3.0791$ & 0.91 \\
\hline $3 p$ & 5.10 & $y=2.8944 x+2.9508$ & 0.94 \\
\hline Lead compound & 12.61 & $y=0.7568 x+4.1669$ & 0.94 \\
\hline $\mathbf{A}$ & 24.21 & $y=1.1892 x+3.3541$ & 0.98 \\
\hline $\mathbf{P}$ & 60.40 & $y=1.0606 x+3.1111$ & 0.92 \\
\hline 嘧菌酯 & 5.17 & $y=0.5026 x+4.6425$ & 0.91 \\
\hline
\end{tabular}

表 5 部分目标化合物对瓜果腐霉病离体抑菌活性 $\mathrm{EC}_{50}$

Table $5 \mathrm{EC}_{50}$ of some target compounds against Pythium aphanider-matum in vitro

\begin{tabular}{cccc}
\hline Compd. & $\mathrm{EC}_{50} /\left(\mu \mathrm{g} \cdot \mathrm{mL}^{-1}\right)$ & 线性方程 & $\mathrm{R}^{2}$ \\
\hline $\mathbf{3 a}$ & 9.04 & $y=2.1430 x+2.9500$ & 0.93 \\
$\mathbf{3 c}$ & 35.15 & $y=1.3196 x+2.9617$ & 0.98 \\
$\mathbf{3 d}$ & 4.35 & $y=1.2912 x+4.1757$ & 0.94 \\
$\mathbf{3 e}$ & 23.72 & $y=2.1148 x+2.0921$ & 0.97 \\
$\mathbf{3 f}$ & 26.43 & $y=2.9907 x+0.7485$ & 0.92 \\
$\mathbf{3 h}$ & 22.21 & $y=3.0688 x+0.8690$ & 0.95 \\
$\mathbf{3 i}$ & 10.12 & $y=1.0758 x+3.9186$ & 0.91 \\
$\mathbf{3 j}$ & 18.27 & $y=1.5940 x+2.9888$ & 0.97 \\
$\mathbf{3} \mathbf{3}$ & 21.65 & $y=2.6595 x+1.4494$ & 0.98 \\
$\mathbf{3 m}$ & 17.72 & $y=2.2467 x+2.1962$ & 0.94 \\
$\mathbf{3 n}$ & 10.49 & $y=1.5598 x+3.1988$ & 0.99 \\
$\mathbf{3 p}$ & 8.40 & $y=4.9150 x+0.4688$ & 0.92 \\
Lead compound & 5.37 & $y=2.3678 x+3.2722$ & 0.90 \\
$\mathbf{A}$ & $\mathbf{5 0 . 0 0}$ & $\boldsymbol{y}=\mathbf{1 . 0 5 0 1} \boldsymbol{x}+\mathbf{3 . 2 1 4 0}$ & $\mathbf{0 . 9 2}$ \\
$\mathbf{P}$ & $\mathbf{1 8 9 . 6 0}$ & $\boldsymbol{y}=\mathbf{0 . 6 9 9 4 x + 3 . 4 0 6 9}$ & $\mathbf{0 . 9 3}$ \\
嘧菌酯 & 16.88 & $y=0.3656 x+4.5528$ & 0.92 \\
\hline
\end{tabular}<smiles>S=C(N/N=C/C=C/c1ccc(Cl)cc1)N1CCCCC1</smiles>

3a<smiles>S=C(N/N=C/c1ccc(Cl)cc1)N1CCCCC1</smiles><smiles>S=C(N/N=C/C=C/c1ccccc1)N1CCCCC1</smiles><smiles>S=C(N/N=C/c1ccccc1)N1CCCCC1</smiles>

图 4 化合物 3a, 3p, A, P 的结构式

Figure 4 Chemical structures of compounds $3 \mathbf{a}, \mathbf{3 p}, \mathbf{A}, \mathbf{P}$

表现出广谱的杀菌活性. 初步构效关系分析表明, 将苯 甲醛骨架跃迁为具有杀菌活性的天然肉桂醛骨架结构, 有利于活性的提高, 值得进一步研究.

\section{3 实验部分}

\section{1 仪器与试剂}

Fukai 显微熔点仪(温度计未校正); Bruker DPX 300 $\mathrm{MHz}$ 核磁共振仪(以 TMS 为内标, DMSO 为溶剂); Vario

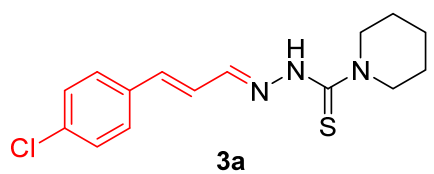

水稻纹枯: $\mathrm{EC}_{50}=2.35 \mu \mathrm{g} / \mathrm{mL}$ 苹果腐烂: $\mathrm{EC}_{50}=5.53 \mu \mathrm{g} / \mathrm{mL}$

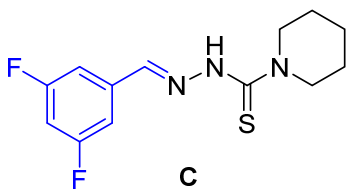

水稻纹枯: $\mathrm{EC}_{50}=11.6 \mu \mathrm{g} / \mathrm{mL}$ 苹果腐烂: $\mathrm{EC}_{50}=12.2 \mu \mathrm{g} / \mathrm{mL}$<smiles>[B]=C(N/N=C/c1c(Cl)cccc1Cl)N1CCCCC1</smiles>

水稻纹枯: $\mathrm{EC}_{50}=15.2 \mu \mathrm{g} / \mathrm{mL}$ 苹果腐烂: $\mathrm{EC}_{50}=10.5 \mu \mathrm{g} / \mathrm{mL}$<smiles>Fc1cccc(/C=N/NC(=S)N2CCCCC2)c1</smiles>

水稻纹枯: $\mathrm{EC}_{50}=8.4 \mu \mathrm{g} / \mathrm{mL}$ 苹果腐烂: $\mathrm{EC}_{50}=18.1 \mu \mathrm{g} / \mathrm{mL}$
图 5 化合物 3a, B, C 和 D 的结构式及生物活性

Figure 5 Chemical structures and fungicidal activities of compounds $\mathbf{3 a}, \mathbf{B}, \mathbf{C}$ and $\mathbf{D}$

EL III 型元素分析仪; Bruker APEX IV 高分辨质谱仪; 柱层析硅胶(200～300 目)和薄层板均为青岛海洋化工有 限公司生产. 所用试剂均为市售分析纯或化学纯试剂, 除特别注明外, 所用溶剂未经无水处理. 


\section{2 实验方法}

\subsection{1 中间体的合成}

(1H-咪唑-1-基)(哌啶-1-基)甲硫酮(1a $\sim 1 \mathbf{p})$ 和哌啶1-硫代碳酰肼 $(2 \mathbf{a} \sim 2 p)$ 按照文献[18]的方法制备, 表征 数据与文献一致.

\subsection{2 目标化合物 $\mathbf{3 a} \sim 3 \mathbf{p}$ 的合成}

将 $3.4 \mathrm{mmol}$ 取代肉桂醛、3.1 $\mathrm{mmol}$ 中间体 $2 、 15 \mathrm{~mL}$ 无水乙醇加入 $50 \mathrm{~mL}$ 三口瓶中, 室温摚拌, $0.5 \mathrm{~h}$ 后溶液 变浑浊, 有固体产生. 通过薄层色谱监测, 待中间体 $\mathbf{2}$ 反应完全后, 停止反应, 反应液过滤后收集固体, 粗产 品经过重结晶 $[V$ (乙醇) $: V$ (正己烷 $)=1 ： 2$ ]纯化, 烘干 即得目标产物.

$N^{\prime}$-[(1E,2E)-3-(4-氯苯基)烯丙基]哌啶-1-硫代酰肼 (3a): 黄色固体, 产率 93\%. m.p. 167.4 168.1 ${ }^{\circ} \mathrm{C} ;{ }^{1} \mathrm{H}$ NMR (300 MHz, DMSO- $\left.d_{6}\right) \delta$ : $10.89(\mathrm{~s}, 1 \mathrm{H}), 7.95$ (t, $J=$ $4.4 \mathrm{~Hz}, 1 \mathrm{H}), 7.64(\mathrm{~d}, J=8.6 \mathrm{~Hz}, 2 \mathrm{H}), 7.42(\mathrm{~d}, J=8.6 \mathrm{~Hz}$, 2H), 6.94 (d, $J=4.4 \mathrm{~Hz}, 2 \mathrm{H}), 3.83$ (d, $J=5.3 \mathrm{~Hz}, 4 \mathrm{H}), 1.56$ (d, $J=3.2 \mathrm{~Hz}, 6 \mathrm{H}) ;{ }^{13} \mathrm{C}$ NMR $\left(75 \mathrm{MHz}\right.$, DMSO- $\left.d_{6}\right) \delta$ : $180.04,145.71,136.48,135.16,133.04,128.89,128.72$, 126.53, 51.08, 25.79, 24.01; IR (KBr) v: 3126, 3040, 1624, $1593,1532,1504,1246,810 \mathrm{~cm}^{-1}$. Anal. calcd for $\mathrm{C}_{15} \mathrm{H}_{18^{-}}$ $\mathrm{ClN}_{3} \mathrm{~S}$ : C 58.53, H 5.89, N 13.65; found C 58.38, H 5.76, $\mathrm{N} 13.56$.

$N^{\prime}$-[(1E,2E)-3-(2-甲基苯基)烯丙基]哌啶-1-硫代酰 肼(3b): 黄色固体, 产率 75\%. m.p. 115.8 116.2 ${ }^{\circ} \mathrm{C} ;{ }^{1} \mathrm{H}$ NMR (300 MHz, DMSO- $\left.d_{6}\right) \delta: 10.93(\mathrm{~s}, 1 \mathrm{H}), 7.90(\mathrm{~s}, 1 \mathrm{H})$, $7.50 \sim 7.35(\mathrm{~m}, 4 \mathrm{H}), 7.33 \sim 7.28(\mathrm{~m}, 1 \mathrm{H}), 6.74(\mathrm{~s}, 1 \mathrm{H})$, $3.83(\mathrm{~d}, J=5.5 \mathrm{~Hz}, 4 \mathrm{H}), 2.06(\mathrm{~d}, J=0.9 \mathrm{~Hz}, 3 \mathrm{H}), 1.61$ (s, $6 \mathrm{H}) ;{ }^{13} \mathrm{C}$ NMR (75 MHz, DMSO- $\left.d_{6}\right) \delta$ : 180.32, 148.42, $136.54,135.99,134.39,129.34,128.52,127.63,51.55$, 25.92, 24.06, 12.85; IR (KBr) v: 3145, 3037, 1617, 1594, $1574,1501,1243,753 \mathrm{~cm}^{-1}$. Anal. calcd for $\mathrm{C}_{16} \mathrm{H}_{21} \mathrm{~N}_{3} \mathrm{~S}: \mathrm{C}$ 66.86, H 7.36, N 14.62; found C 66.81, H 7.23, N 14.55.

$N^{\prime}$-[(1E,2E)-3-(2-甲氧基苯基)烯丙基]哌啶-1-硫代 酰胇 $(3 \mathrm{c})$ : 白色固体, 产率 $69 \%$. m.p. $154.6 \sim 155.6{ }^{\circ} \mathrm{C}$; ${ }^{1} \mathrm{H}$ NMR (300 MHz, DMSO- $\left.d_{6}\right) \delta$ : $10.87(\mathrm{~s}, 1 \mathrm{H}), 7.95$ (d, $J=9.1 \mathrm{~Hz}, 1 \mathrm{H}), 7.63(\mathrm{~d}, J=6.3 \mathrm{~Hz}, 1 \mathrm{H}), 7.33 \sim 7.28(\mathrm{~m}$, $1 \mathrm{H}), 7.16 \sim 6.85(\mathrm{~m}, 4 \mathrm{H}), 3.98 \sim 3.75(\mathrm{~m}, 7 \mathrm{H}), 1.58(\mathrm{~s}$, $6 \mathrm{H}) ;{ }^{13} \mathrm{C}$ NMR (75 MHz, DMSO- $\left.d_{6}\right) \delta$ : 180.00, 156.85, $146.72,132.81,130.14,127.35,126.14,124.45,120.83$, 111.66, 55.68, 51.09, 25.78, 24.03; IR (KBr) v: 3149, $3035,1619,1598,1575,1487,1243,781 \mathrm{~cm}^{-1}$. Anal. calcd for $\mathrm{C}_{16} \mathrm{H}_{21} \mathrm{~N}_{3} \mathrm{OS}$ : C 63.34, H 6.98, N 13.85; found C 63.07, H 6.91, N 13.97 .

$N^{N}$-[(1E,2E)-3-(4-甲氧基苯基)烯丙基]哌啶-1-硫代 酰肼(3d): 黄色固体, 产率 $73 \%$. m.p. $117.4 \sim 118.6{ }^{\circ} \mathrm{C}$; ${ }^{1} \mathrm{H}$ NMR $\left(300 \mathrm{MHz}\right.$, DMSO- $\left.d_{6}\right) \delta: 10.83(\mathrm{~s}, 1 \mathrm{H}), 7.94(\mathrm{~d}$, $J=8.9 \mathrm{~Hz}, 1 \mathrm{H}), 7.53(\mathrm{~d}, J=8.8 \mathrm{~Hz}, 2 \mathrm{H}), 6.97 \sim 6.70(\mathrm{~m}$, $4 \mathrm{H}), 3.83 \sim 3.79(\mathrm{~m}, 4 \mathrm{H}), 3.77(\mathrm{~s}, 3 \mathrm{H}), 1.65 \sim 1.51(\mathrm{~m}$, $6 \mathrm{H}) ;{ }^{13} \mathrm{C}$ NMR (75 MHz, DMSO- $\left.d_{6}\right) \delta: 179.95,159.87$, $146.51,137.92,128.86,128.57,123.32,114.40,55.34$, 51.04, 25.79, 24.04; IR (KBr) v: 3131, 2943, 1596, 1535, 1500, 1340, 1247, $745 \mathrm{~cm}^{-1}$; HRMS (ESI) calcd for $\mathrm{C}_{15} \mathrm{H}_{19} \mathrm{~N}_{4} \mathrm{O}_{2} \mathrm{~S}\left([\mathrm{M}+\mathrm{H}]^{+}\right)$319.1223, found 319.1226.

$N^{\prime}$-[(1E,2E)-3-(4-硝基苯基)烯丙基]哌啶-1-硫代酰 肼(3e): 黄色固体, 产率 $80 \%$. m.p. $168.2 \sim 169.5{ }^{\circ} \mathrm{C} ;{ }^{1} \mathrm{H}$ NMR (300 MHz, DMSO- $\left.d_{6}\right) \delta$ : $11.05(\mathrm{~s}, 1 \mathrm{H}), 8.18$ (d, $J=$ $8.8 \mathrm{~Hz}, 2 \mathrm{H}), 8.00(\mathrm{~d}, J=8.4 \mathrm{~Hz}, 1 \mathrm{H}), 7.84(\mathrm{~d}, J=8.8 \mathrm{~Hz}$, $2 \mathrm{H}), 7.21 \sim 7.00(\mathrm{~m}, 2 \mathrm{H}), 3.84(\mathrm{~d}, J=5.4 \mathrm{~Hz}, 4 \mathrm{H}), 1.59(\mathrm{~d}$, $J=6.1 \mathrm{~Hz}, 6 \mathrm{H}) ;{ }^{13} \mathrm{C}$ NMR $\left(75 \mathrm{MHz}\right.$, DMSO- $\left.d_{6}\right) \delta: 180.04$, $146.78,145.03,142.92,135.18,130.27,127.92,124.06$, 51.12, 25.79, 23.99; IR (KBr) v: 3142, 2937, 1604, 1576, 1497, 1238, 1173, $819 \mathrm{~cm}^{-1}$; HRMS (ESI) calcd for $\mathrm{C}_{16} \mathrm{H}_{22} \mathrm{~N}_{3} \mathrm{OS}\left([\mathrm{M}+\mathrm{H}]^{+}\right)$304.1478, found 304.1481.

乙基 1-(2-((1E,2E)-3-(4-氯苯基)烯丙基)酰肼-1-碳硫 代酯) 哌啶-4-羧酸酯 (3f): 白色固体, 产率 $73 \%$. m.p. $127.2 \sim 128.0{ }^{\circ} \mathrm{C} ;{ }^{1} \mathrm{H}$ NMR (300 MHz, DMSO- $\left.d_{6}\right) \delta$ : $11.06(\mathrm{~s}, 1 \mathrm{H}), 8.04 \sim 7.87(\mathrm{~m}, 1 \mathrm{H}), 7.65(\mathrm{~d}, J=8.6 \mathrm{~Hz}$, $2 \mathrm{H}), 7.42(\mathrm{~d}, J=8.5 \mathrm{~Hz}, 2 \mathrm{H}), 6.96(\mathrm{~d}, J=4.8 \mathrm{~Hz}, 2 \mathrm{H}), 4.50$ (d, $J=13.1 \mathrm{~Hz}, 2 \mathrm{H}), 4.09$ (q, $J=7.1 \mathrm{~Hz}, 2 \mathrm{H}), 3.24$ (t, $J=$ $11.3 \mathrm{~Hz}, 2 \mathrm{H}), 2.77 \sim 2.60(\mathrm{~m}, 1 \mathrm{H}), 1.89(\mathrm{~d}, J=10.4 \mathrm{~Hz}$, $2 \mathrm{H}), 1.66 \sim 1.55(\mathrm{~m}, 2 \mathrm{H}), 1.20(\mathrm{t}, J=7.1 \mathrm{~Hz}, 3 \mathrm{H}) ;{ }^{13} \mathrm{C}$ NMR $\left(75 \mathrm{MHz}\right.$, DMSO- $\left.d_{6}\right) \delta$ : $180.42,173.96,146.04$, $136.81,135.12,133.10,128.87,128.77,126.45,60.12$, 49.27, 40.06, 28.01, 14.20; IR (KBr) v: 3123, 1732, 1625, $1593,1536,1489,1249,807 \mathrm{~cm}^{-1}$; HRMS (ESI) calcd for $\mathrm{C}_{18} \mathrm{H}_{23} \mathrm{ClN}_{3} \mathrm{O}_{2} \mathrm{~S}\left([\mathrm{M}+\mathrm{H}]^{+}\right) 380.1194$, found 380.1192 .

乙基-1-(2-((1E,2E)-3-(2-甲基苯基)烯丙基)酰肼-1碳硫代酯)哌啶-4-羧酸酯 $(\mathbf{g g})$ ：白色固体，产率 $63 \%$. m.p. $136.0 \sim 138.0{ }^{\circ} \mathrm{C} ;{ }^{1} \mathrm{H}$ NMR (300 MHz, DMSO- $\left.d_{6}\right) \delta$ : 11.05 (s, 1H), $7.91(\mathrm{~s}, 1 \mathrm{H}), 7.47 \sim 7.38(\mathrm{~m}, 4 \mathrm{H}), 7.31(\mathrm{~d}$, $J=7.0 \mathrm{~Hz}, 1 \mathrm{H}), 6.76(\mathrm{~s}, 1 \mathrm{H}), 4.51(\mathrm{~d}, J=13.2 \mathrm{~Hz}, 2 \mathrm{H})$, 4.09 (q, $J=7.1 \mathrm{~Hz}, 2 \mathrm{H}), 3.25$ (t, $J=11.1 \mathrm{~Hz}, 2 \mathrm{H}), 2.79 \sim$ $2.59(\mathrm{~m}, 1 \mathrm{H}), 2.06(\mathrm{~s}, 3 \mathrm{H}), 1.90(\mathrm{~d}, J=10.4 \mathrm{~Hz}, 2 \mathrm{H})$, $1.68 \sim 1.57(\mathrm{~m}, 2 \mathrm{H}), 1.18(\mathrm{t}, J=7.1 \mathrm{~Hz}, 3 \mathrm{H}) ;{ }^{13} \mathrm{C}$ NMR $(75$ MHz，DMSO- $\left.d_{6}\right) \quad \delta: 180.67,173.96,148.79,136.50$, $136.26,134.32,129.37,128.53,127.68,60.11,49.62$, 40.09, 28.14, 14.19, 12.85; IR (KBr) v: 3157, 3027, 1725, $1614,1596,1500,1208,756 \mathrm{~cm}^{-1}$. Anal. calcd for $\mathrm{C}_{19} \mathrm{H}_{25^{-}}$ $\mathrm{N}_{3} \mathrm{O}_{2} \mathrm{~S}$ : C 63.48, H 7.01, N 11.69; found C 63.47, H 6.91, 
N 11.73.

乙基-1-(2-((1E,2E)-3-(2-甲氧基苯基)烯丙基)酰肼1-碳硫代酯)哌啶-4-羧酸酯(3h): 白色固体, 产率 $59 \%$. m.p. $152.8 \sim 153.5{ }^{\circ} \mathrm{C} ;{ }^{1} \mathrm{H}$ NMR (300 MHz, DMSO- $d_{6}$ ) $\delta$ : $10.98(\mathrm{~s}, 1 \mathrm{H}), 7.95(\mathrm{~d}, J=9.2 \mathrm{~Hz}, 1 \mathrm{H}), 7.65 \sim 7.62(\mathrm{~m}$, $1 \mathrm{H}), 7.39 \sim 7.24(\mathrm{~m}, 1 \mathrm{H}), 7.17 \sim 6.90(\mathrm{~m}, 4 \mathrm{H}), 4.50(\mathrm{~d}, J=$ $13.3 \mathrm{~Hz}, 2 \mathrm{H}), 4.09$ (q, $J=7.1 \mathrm{~Hz}, 2 \mathrm{H}), 3.85$ (s, 3H), 3.24 (t, $J=11.2 \mathrm{~Hz}, 2 \mathrm{H}), 2.81 \sim 2.57(\mathrm{~m}, 1 \mathrm{H}), 1.88(\mathrm{~d}, J=10.4$ $\mathrm{Hz}, 2 \mathrm{H}), 1.76 \sim 1.45$ (m, 2H), 1.19 (t, $J=7.1 \mathrm{~Hz}, 3 \mathrm{H}) ;{ }^{13} \mathrm{C}$ NMR (75 MHz, DMSO- $\left.d_{6}\right) \delta$ : 180.38, 173.96, 156.86, $147.03,133.08,130.21,127.37,126.03,124.41,120.82$, 111.67, 60.12, 55.68, 49.27, 40.08, 28.01, 14.20; IR (KBr) v: 3141, 1733, 1616, 1596, 1539, 1486, 1248, $755 \mathrm{~cm}^{-1}$. Anal. calcd for $\mathrm{C}_{19} \mathrm{H}_{25} \mathrm{~N}_{3} \mathrm{O}_{3} \mathrm{~S}$ : C 60.78, H 6.71, N 11.19; found $\mathrm{C} 60.50, \mathrm{H} 6.62, \mathrm{~N} 11.10$.

乙基-1-(2-((1E,2E)-3-(4-甲氧基苯基)烯丙基)酰肼1-碳硫代酯) 哌啶-4-羧酸酯(3i): 黄色固体, 产率 $64 \%$. m.p. 126.7 $128.2{ }^{\circ} \mathrm{C} ;{ }^{1} \mathrm{H}$ NMR (300 MHz, DMSO- $d_{6}$ ) $\delta$ : $10.94(\mathrm{~s}, 1 \mathrm{H}), 7.93(\mathrm{~d}, J=8.9 \mathrm{~Hz}, 1 \mathrm{H}), 7.54(\mathrm{~d}, J=8.8 \mathrm{~Hz}$, $2 \mathrm{H}), 6.94 \sim 6.73(\mathrm{~m}, 4 \mathrm{H}), 4.50(\mathrm{~d}, J=13.2 \mathrm{~Hz}, 2 \mathrm{H}), 4.08$ (q, $J=7.1 \mathrm{~Hz}, 2 \mathrm{H}), 3.77(\mathrm{~s}, 3 \mathrm{H}), 3.26 \sim 3.19(\mathrm{~m}, 2 \mathrm{H})$, $2.73 \sim 2.61(\mathrm{~m}, 1 \mathrm{H}), 1.91 \sim 1.85(\mathrm{~m}, 2 \mathrm{H}), 1.67 \sim 1.54(\mathrm{~m}$, 2H), $1.19(\mathrm{t}, J=7.1 \mathrm{~Hz}, 3 \mathrm{H}) ;{ }^{13} \mathrm{C}$ NMR $(75 \mathrm{MHz}$, DMSO- $\left.d_{6}\right) \delta: 180.32,173.98,159.91,146.84,138.25$, 128.83, 128.63, 123.24, 114.39, 60.12, 55.34, 49.23, 40.09, 28.01, 14.20; IR (KBr) v: 3137, 2978, 1732, 1718, 1595, $1509,1336,744 \mathrm{~cm}^{-1}$; HRMS (ESI) calcd for $\mathrm{C}_{18} \mathrm{H}_{23} \mathrm{~N}_{4}-$ $\mathrm{O}_{4} \mathrm{~S}\left([\mathrm{M}+\mathrm{H}]^{+}\right)$391.1435, found 391.1436.

乙基-1-(2-((1E,2E)-3-(4-硝基苯基)烯丙基)酰肼-1碳硫代酯)哌啶-4-羒酸酯(3j): 黄色固体, 产率 54\%. m.p. $124.6 \sim 125.8{ }^{\circ} \mathrm{C} ;{ }^{1} \mathrm{H}$ NMR (300 MHz, DMSO- $d_{6}$ ) $\delta$ : $11.17(\mathrm{~s}, 1 \mathrm{H}), 8.19$ (d, $J=8.9 \mathrm{~Hz}, 2 \mathrm{H}), 8.00(\mathrm{~d}, J=8.3 \mathrm{~Hz}$, $1 \mathrm{H}), 7.86(\mathrm{~d}, J=8.9 \mathrm{~Hz}, 2 \mathrm{H}), 7.21 \sim 7.04(\mathrm{~m}, 2 \mathrm{H}), 4.50(\mathrm{~d}$, $J=13.1 \mathrm{~Hz}, 2 \mathrm{H}), 4.08$ (q, $J=7.1 \mathrm{~Hz}, 2 \mathrm{H}), 3.27$ (t, $J=11.2$ $\mathrm{Hz}, 2 \mathrm{H}), 2.72 \sim 2.64(\mathrm{~m}, 1 \mathrm{H}), 1.89(\mathrm{~d}, J=10.3 \mathrm{~Hz}, 2 \mathrm{H})$, $1.68 \sim 1.54(\mathrm{~m}, 2 \mathrm{H}), 1.19(\mathrm{t}, J=7.1 \mathrm{~Hz}, 3 \mathrm{H}) ;{ }^{13} \mathrm{C} \mathrm{NMR}(75$ $\mathrm{MHz}$, DMSO- $\left.d_{6}\right) \delta$ : $180.45,173.94,146.83,145.36$, $142.88,135.51,130.19,127.98,124.07,60.14,49.30$, 40.03, 28.01, 14.20; IR (KBr) v: 3121, 2955, 1717, 1603, 1508, 1498, 1198, $821 \mathrm{~cm}^{-1}$; HRMS (ESI) calcd for $\mathrm{C}_{19} \mathrm{H}_{26} \mathrm{~N}_{3} \mathrm{O}_{3} \mathrm{~S}\left([\mathrm{M}+\mathrm{H}]^{+}\right)$376.1689, found 376.1692.

$N^{\prime}$-((1E,2E)-3-(4-氯苯基)烯丙基)-3-甲基哌啶-1-硫 代酰肼(3k): 黄色固体, 产率 39\%. m.p. 99.0 100.0 ${ }^{\circ} \mathrm{C}$; ${ }^{1} \mathrm{H}$ NMR $\left(300 \mathrm{MHz}\right.$, DMSO- $\left.d_{6}\right) \delta: 10.96(\mathrm{~s}, 1 \mathrm{H}), 8.05 \sim$ 7.90 (m, 1H), 7.64 (d, $J=8.5 \mathrm{~Hz}, 2 \mathrm{H}), 7.42(\mathrm{~d}, J=8.5 \mathrm{~Hz}$,
2H), $7.04 \sim 6.84(\mathrm{~m}, 2 \mathrm{H}), 4.55 \sim 4.45(\mathrm{~m}, 2 \mathrm{H}), 3.06(\mathrm{t}, J=$ $11.0 \mathrm{~Hz}, 1 \mathrm{H}), 2.85 \sim 2.68(\mathrm{~m}, 1 \mathrm{H}), 1.85 \sim 1.39(\mathrm{~m}, 4 \mathrm{H})$, $1.31 \sim 1.01(\mathrm{~m}, 1 \mathrm{H}), 0.88 \sim 0.86(\mathrm{~m}, 3 \mathrm{H}) ;{ }^{13} \mathrm{C}$ NMR $(75$ MHz, DMSO- $\left.d_{6}\right) \delta: 179.98,145.78,136.52,135.14$, 133.06, 128.90, 128.74, 126.49, 57.03, 50.57, 32.54, 31.17, $25.05,18.84$; IR (KBr) v: 3123, 1732, 1625, 1593, 1536, 1489, 1249, $807 \mathrm{~cm}^{-1}$; HRMS (ESI) calcd for $\mathrm{C}_{16} \mathrm{H}_{21^{-}}$ $\mathrm{ClN}_{3} \mathrm{~S}\left([\mathrm{M}+\mathrm{H}]^{+}\right) 322.1139$, found 322.1138 .

$N^{\prime}$-((1E,2E)-3-(2-甲基苯基)烯丙基)-3-甲基哌啶-1硫代酰肼 (3I): 黄色固体, 产率 60\%. m.p. 130.8 $131.8{ }^{\circ} \mathrm{C} ;{ }^{1} \mathrm{H}$ NMR $\left(300 \mathrm{MHz}\right.$, DMSO- $\left.d_{6}\right) \delta: 10.94$ (s, $1 \mathrm{H}), 7.91(\mathrm{~s}, 1 \mathrm{H}), 7.51 \sim 7.36(\mathrm{~m}, 4 \mathrm{H}), 7.35 \sim 7.25(\mathrm{~m}$, $1 \mathrm{H}), 6.75(\mathrm{~s}, 1 \mathrm{H}), 4.59 \sim 4.48(\mathrm{~m}, 2 \mathrm{H}), 3.08 \sim 2.99(\mathrm{~m}$, $1 \mathrm{H}), 2.78 \sim 2.70(\mathrm{~m}, 1 \mathrm{H}), 2.06(\mathrm{~d}, J=0.9 \mathrm{~Hz}, 3 \mathrm{H}), 1.87 \sim$ $1.39(\mathrm{~m}, 4 \mathrm{H}), 1.24 \sim 1.11(\mathrm{~m}, 1 \mathrm{H}), 0.87(\mathrm{~d}, J=6.6 \mathrm{~Hz}$, $3 \mathrm{H}) ;{ }^{13} \mathrm{C}$ NMR (75 MHz, DMSO- $d_{6}$ ) $\delta: 180.20,148.47$, $136.52,136.05,134.34,129.35,128.53,127.65,57.50$, $51.09,32.59,31.28,25.26,18.82,12.80$; IR (KBr) v: 3157, $3028,1615,1597,1503,1243,758 \mathrm{~cm}^{-1}$. Anal. calcd for $\mathrm{C}_{17} \mathrm{H}_{23} \mathrm{~N}_{3} \mathrm{~S}$ : C 67.73, H 5.69, N 13.94; found C 67.77, H 5.78, N 13.93.

$N^{\prime}$-((1E,2E)-3-(2-甲氧基苯基)烯丙基)-3-甲基哌啶1-硫代酰肼(3m): 黄色固体, 产率 $40 \%$. m.p. $76 \sim 78{ }^{\circ} \mathrm{C}$; ${ }^{1} \mathrm{H}$ NMR (300 MHz, DMSO- $\left.d_{6}\right) \delta: 10.87(\mathrm{~s}, 1 \mathrm{H}), 7.95(\mathrm{~d}$, $J=9.2 \mathrm{~Hz}, 1 \mathrm{H}), 7.63(\mathrm{~d}, J=6.6 \mathrm{~Hz}, 1 \mathrm{H}), 7.34 \sim 7.29(\mathrm{~m}$, $1 \mathrm{H}), 6.98 \sim 6.88(\mathrm{~m}, 4 \mathrm{H}), 4.50(\mathrm{t}, J=13.6 \mathrm{~Hz}, 2 \mathrm{H}), 3.85(\mathrm{~s}$, $3 \mathrm{H}), 3.10 \sim 3.02(\mathrm{~m}, 1 \mathrm{H}), 2.83 \sim 2.71(\mathrm{~m}, 1 \mathrm{H}), 1.84 \sim 1.45$ (m, $4 \mathrm{H}), 1.28 \sim 1.13(\mathrm{~m}, 1 \mathrm{H}), 0.88(\mathrm{~d}, J=6.6 \mathrm{~Hz}, 3 \mathrm{H}) ;{ }^{13} \mathrm{C}$ NMR $\left(75 \mathrm{MHz}\right.$, DMSO- $\left.d_{6}\right) \delta$ : 179.93, 156.87, 146.76, $132.85,130.15,127.40,126.15,124.45,120.83,111.67$, $57.08,55.69,50.61,32.55,31.18,25.05,18.81$; IR (KBr) $v: 3165,3042,1616,1597,1548,1456,1239,749 \mathrm{~cm}^{-1}$. Anal. calcd for $\mathrm{C}_{17} \mathrm{H}_{23} \mathrm{~N}_{3} \mathrm{OS}$ : C 64.32, H 7.30, N 13.24; found $\mathrm{C} 64.15, \mathrm{H} 7.33, \mathrm{~N} 13.01$.

$N^{\prime}$-((1E,2E)-3-(4-甲氧基苯基)烯丙基)-3-甲基哌啶1-硫代酰肼(3n): 黄色固体, 产率 49\%. m.p. 92.1 $93.7{ }^{\circ} \mathrm{C} ;{ }^{1} \mathrm{H}$ NMR $\left(300 \mathrm{MHz}\right.$, DMSO- $\left.d_{6}\right) \delta: 10.85(\mathrm{~s}, 1 \mathrm{H})$, $7.95(\mathrm{~d}, J=8.9 \mathrm{~Hz}, 1 \mathrm{H}), 7.53$ (d, $J=8.8 \mathrm{~Hz}, 2 \mathrm{H}), 6.96 \sim$ $6.72(\mathrm{~m}, 4 \mathrm{H}), 4.50(\mathrm{t}, J=13.2 \mathrm{~Hz}, 2 \mathrm{H}), 3.76(\mathrm{~s}, 3 \mathrm{H})$, $3.12 \sim 2.96(\mathrm{~m}, 1 \mathrm{H}), 2.79 \sim 2.71(\mathrm{~m}, 1 \mathrm{H}), 1.81 \sim 1.59(\mathrm{~m}$, $3 \mathrm{H}), 1.54 \sim 1.42(\mathrm{~m}, 1 \mathrm{H}), 1.22 \sim 1.09(\mathrm{~m}, 1 \mathrm{H}), 0.86(\mathrm{~d}, J=$ $6.6 \mathrm{~Hz}, 3 \mathrm{H}) ;{ }^{13} \mathrm{C}$ NMR $\left(75 \mathrm{MHz}\right.$, DMSO- $\left.d_{6}\right) \delta$ : 179.91, $159.88,146.55,137.94,128.85,128.58,123.30,114.40$, 57.02, 55.34, 50.54, 32.57, 31.16, 25.05, 18.83; IR (KBr) v: 3132, 2964, 1732, 1716, 1594, 1511, 1335, $745 \mathrm{~cm}^{-1}$; 
HRMS (ESI) calcd for $\mathrm{C}_{16} \mathrm{H}_{21} \mathrm{~N}_{4} \mathrm{O}_{2} \mathrm{~S}\left([\mathrm{M}+\mathrm{H}]^{+}\right) 333.1380$, found 333.1382

$N^{\prime}$-((1E,2E)-3-(4-硝基苯基)烯丙基)-3-甲基哌啶-1硫代酰肼(3o): 红色固体，产率 52\%. m.p. 153.8 $155.0{ }^{\circ} \mathrm{C}$; ${ }^{1} \mathrm{H}$ NMR (300 MHz, DMSO- $\left.d_{6}\right) \delta$ : 11.06 (s, $1 \mathrm{H}), 8.17(\mathrm{~d}, J=8.8 \mathrm{~Hz}, 2 \mathrm{H}), 8.00(\mathrm{~d}, J=8.2 \mathrm{~Hz}, 1 \mathrm{H}), 7.85$ $(\mathrm{d}, J=8.9 \mathrm{~Hz}, 2 \mathrm{H}), 7.26 \sim 6.97(\mathrm{~m}, 2 \mathrm{H}), 4.45(\mathrm{t}, J=12.6$ $\mathrm{Hz}, 2 \mathrm{H}), 3.17 \sim 2.98(\mathrm{~m}, 1 \mathrm{H}), 2.83 \sim 2.75(\mathrm{~m}, 1 \mathrm{H}), 1.84 \sim$ $1.58(\mathrm{~m}, 3 \mathrm{H}), 1.55 \sim 1.43(\mathrm{~m}, 1 \mathrm{H}), 1.24 \sim 1.11(\mathrm{~m}, 1 \mathrm{H})$, $0.87(\mathrm{~d}, J=6.6 \mathrm{~Hz}, 3 \mathrm{H}) ;{ }^{13} \mathrm{C} \mathrm{NMR}\left(75 \mathrm{MHz}, \mathrm{DMSO}-d_{6}\right) \delta$ : $180.01,146.79,145.10,142.92,135.21,130.24,127.93$, $124.07,57.07,50.63,32.51,31.19,25.04,18.82$; IR (KBr) v: $3169,3043,1734,1602,1508,1238,1173,819 \mathrm{~cm}^{-1}$; HRMS (ESI) calcd for $\mathrm{C}_{17} \mathrm{H}_{24} \mathrm{~N}_{3} \mathrm{OS}\left([\mathrm{M}+\mathrm{H}]^{+}\right)$318.1635, found 318.1646 .

\section{3 生物活性测定}

采用菌丝生长速率法测定目标化合物对病原真菌 的抑制率 ${ }^{[19]}$. 供试菌种分别为瓜果腐霉病菌(Pythium aphanider-matum)、水稻纹枯病菌(Rhizoctonia solani)、 苹果腐烂病菌(Valsa mali)、油菜菌核病菌(Sclerotinia sclerotiorum)、水稻恶苗病菌 (Fusarium moniliforme Sheld). 嘧菌酯和吡唑醚菌酯是目前市场上最好的两个 广谱性杀菌剂, 来源于天然产物的改造, 因此选择这两 个商品化杀菌剂作为阳性对照

将化合物溶于二甲基亚砜，配制成 $10000 \mu \mathrm{g} / \mathrm{mL}$ 的 药液备用. 取 $1 \mathrm{~mL}$ 备用药液与 $199 \mathrm{~mL}$ PDA 培养基混 匀, 制备成浓度为 $50 \mu \mathrm{g} / \mathrm{mL}$ 的含毒培养基. 将其倒入直 径为 $90 \mathrm{~cm}$ 的培养血中, 每皿大约 $25 \mathrm{~mL}$, 待其固化后, 接入活化好的病原真菌菌饼, 置于 $25{ }^{\circ} \mathrm{C}$ 培养箱中培养. 二甲基亚砜为溶剂对照, 无菌水为空白对照, 每个样品 重复 3 次. 以上操作为严格的无菌操作, 待空白对照菌 落充分生长后, 以十字交叉法量取菌落直径, 取其平均 值. 按照以下公式计算菌丝生长抑制率:

抑制率 $(\%)=($ 对照菌落直径一处理菌落直径 $) /($ 对照 菌落直径－菌饼直径) $\times 100 \%$

抑制中浓度 $\left(\mathrm{EC}_{50}\right)$ 的测定：选取抑制率大于 $80 \%$ 的 化合物测定其抑制中浓度值. 根据上述方法，采用等倍 稀释，分别测试浓度为 $25.00 ， 12.50 ， 6.25 ， 3.12,1.56$, $0.78 \mu \mathrm{g} / \mathrm{mL}$ 时的抑制率, 然后用软件 SPSS 计算抑制中
浓度 $\mathrm{EC}_{50}$.

辅助材料(Supporting Information) 目标化合物 3a 30 的 ${ }^{1} \mathrm{H}$ NMR、 ${ }^{13} \mathrm{C}$ NMR，以及化合物 3p、A、P 的 ${ }^{1} \mathrm{H}$ NMR. 这些材料可以免费从本刊网站(http://sioc-journal. $\mathrm{cn} /)$ 上下载.

\section{References}

[1] Godfray, H. C. J.; Beddington, J. R.; Crute, I. R.; Haddad, L.; Lawrence, D.; Muir, J. F.; Pretty, J.; Robinson, S.; Thomas, S. M.; Toulmin, C. Science 2010, 327, 812.

[2] Gerwick, B. C.; Sparks, T. C. Pest Manage. Sci. 2014, 70, 1169.

[3] Balba, H. J. Environ. Sci. Health, Part B 2007, 42, 441.

[4] Sparks, T. C.; Hahn, D. R.; Garizi, N. V. Pest Manage. Sci. 2017, 73,700 .

[5] Ashakirin, S. N.; Tripathy, M.; Patil, U. K.; Abdul Majeed, A. B. Int. J. Pharm. Sci. Res. 2017, 8, 2333

[6] Ka, H.; Park, H. J.; Jung, H. J.; Choi, J. W.; Cho, K. S.; Ha, J.; Lee, K. T. Cancer Lett. 2003, 196, 143.

[7] Chang, S. T.; Chen, P. F.; Chang, S. C. J. Ethnopharmacol. 2001, $77,123$.

[8] Zhu, Y. J.; Song, K. K.; Li, Z. C.; Pan, Z. Z.; Guo, Y. J.; Zhou, J. J.; Wang, Q.; Liu, B.; Chen, Q. X. J. Agric. Food Chem. 2009, 57, 5518 .

[9] Gutiérrez, L.; Escudero, A.; Batlle, R.; Nerín, C. J. Agric. Food Chem. 2009, 57, 8564.

[10] Du, W. X.; Avena-Bustillos, R. J.; Woods, R.; Breksa, A. P.; McHugh, T. H.; Friedman, M.; Levin, C. E.; Mandrell, R. J. Agric. Food Chem. 2012, 60, 7799 .

[11] Niknahad, H.; Shuhendler, A.; Galati, G.; Siraki, A. G.; Easson, E.; Poon, R.; O'Brien, P. J. Chem. Biol. Interact. 2003, 143, 119.

[12] Cheng, S. S.; Liu, J. Y.; Tsai, K. H.; Chen, W. J.; Chang, S. T. J. Agric. Food Chem. 2004, 52, 4395.

[13] Yu, Y.; Kalinowski, D. S.; Kovacevic, Z.; Siafakas, A. R.; Jansson, P. J.; Stefani, C.; Lovejoy, D. B.; Sharpe, P. C.; Bernhardt, P. V.; Richardson, D. R. J. Med. Chem. 2009, 52, 5271

[14] Dong, H.; Liu, J.; Liu, X.; Yu, Y.; Cao, S. J. Mol. Struct. 2018 $1151,353$.

[15] Dong, H.; Liu, J.; Liu, X.; Yu, Y.; Cao, S. Bioorg. Chem. 2017, 75, 106.

[16] Liu, L. F.; Li, P. Y.; Qian, Q. Q.; Lei, X. L.; Huang, Y. X. Chin. J. Org. Chem. 2013, 33, 854 (in Chinese). (刘利锋，李培源，钱全全，雷晓琳，黄钰湘，周泉，黄珊，肖琦, 苏炜, 有机化学, 2013, 33, 854.)

[17] Xu, Y.; Wang, Z.; Dong, W.; Xing, J.; Liang, P.; Yang, X. L. Chin. J. Org. Chem. 2012, 32, 1278 (in Chinese). (徐炎, 王振, 凌云, 董玮, 邢静, 梁沛, 杨新玲, 有机化学, 2012, 32, 1278.)

[18] Zhang, X. B.; Lei, P.; Sun, T. D; Jin, X. Y.; Yang, X. L.; Ling, Y. Molecules 2017, 22, 2085.

[19] Zhang, X. M.; Lei, P.; Li, X. L.; Yang, X. L.; Zhang, X. B.; Sun, T. D.; Ling, Y. Chin. J. Org. Chem. 2019, 39, 3197 (in Chinese). (张晓鸣, 雷鹏, 李欣潞, 杨新玲, 张学博, 孙腾达, 凌云, 有机 化学, 2019, 39, 3197.) 